\title{
Intensity determination of cavitation at rotating pumps by means of integrated miniaturised pressure transducers
}

\author{
Werner, Reinhard; Werthschützky, Roland \\ TU Darmstadt, Institut for Electromechanical Design \\ Merckstraße 25, D-64283 Darmstadt
}

\begin{abstract}
In this paper we present a method of cavitation detection with miniaturised pressure transducers in rotodynamic pumps. The process is part of a sensor system for pumps and make it possible to detect the operational mode. Cavitation generates surges which are directed by the fluid to the ambiend pipes and casings and hit them. The vibrations at the surface can be measured by accelerators. Thus, the existence of cavitatation is detectable. The idea is now, to detect the cavitation directly by a pressure transducer and use it like a hydrophon. The difference is that pressure transducers already integrated in the pump for pressure measurement, a hydrophon would be an additional element in the system which probably would only be used for this new system functionallity.
\end{abstract}

\section{Introduction}

Rotodynamic pumps transport liquid fluids in industry. Most of time, they work without interruption at constant rotational speed. Normally, their operational mode is unknown and a break down, e.g. due to abrasion, happens unexpectedly. Hence, the research project "Integration of a sensor system in rotodynamic pumps", financed by the German Research Foundation, is focused on the prevention of break downs by integrating sensors into rotodynamic pumps and the analysis of their signals. Furthermore, when the pump runs in a favourable operational mode (no cavitation, high efficiency,...), the life time cycle increases and the maintenance cost decreases. Altogether, the reliability of the whole system should go up. This goal is in contrast to the fact that the whole reliability decreases with the increase of parts in system. In order to prevent the sensor system to break down, when a sensor fails, redundancy is necessary. One possible option is to measure each measurand by several sensors at the same time (hot redundancy). However, this method has a great disadvantage because the required number of sensors and the expense is significantly higher. Another option is the use of analytical redundancy. Some system properties are archived in the control unit and it will be possible to see with a plausibility check, if the measurand are in a correct relation to each other. If the value of one sensor is out of plane over a longer time, its signal will never be considered for calculation in system any longer. In this case, an alternatively calculating design basis is archiving the missing value.

The main element of this functionality is the possibility to get further information out of the sensor signal about the system state. For example, static pressure signals give additional information related to the flow rate in the rotodynamic pump [1] and the dynamic part gives knowledge of the current rotational speed [2]. If the dynamic part of the pressure transducers signal could used for cavitation detection, an accerometer won't be necessary any longer.

\section{Cavitation}

Cavitation is a formation and activity of bubbles in a liquid. Formation means both, the creation of a new cavity and the growth of the such cavity to a bubble which can seen in macroscopic scale. The bubbles can be filled with gas which was dissolved in the liquid or vapour [3]. The main focus in this paper will be the vapor filled bubbes, because of their destructiv properties in hydraulic systems. Now to the chronolonical sequence of cavitation (Fig. 1): It starts with a smal cavity, which is carried by liquid in a area were the ambient pressure decreases, based on hydrodynamic processes, under vapor pressure. So, the bubble growth until it comes to a area where the ambient pressure increases. Now, the vapor condenses and the bubble implodes and surges run through the water. In case of a colapse in the near of a surface the implosion won't be symetricaly. Insteed, the water can't follow from all sides in the same way, the bubble gets a concave area (Fig. $1 b+c)$ and finally a micro jet was formed and shot to the surface (Fig. 1d). The pressure peak at the surface is probably above $10^{4}$ til $10^{5}$ bar [4]. The result of this event is a dent in the material (fig 2). If pump parts are impacted on cavitation over a long time, they will 
be destroied. So, it is one function of the sensor system to detect cavitation, ro change the operational mode and to avoid canvitation.
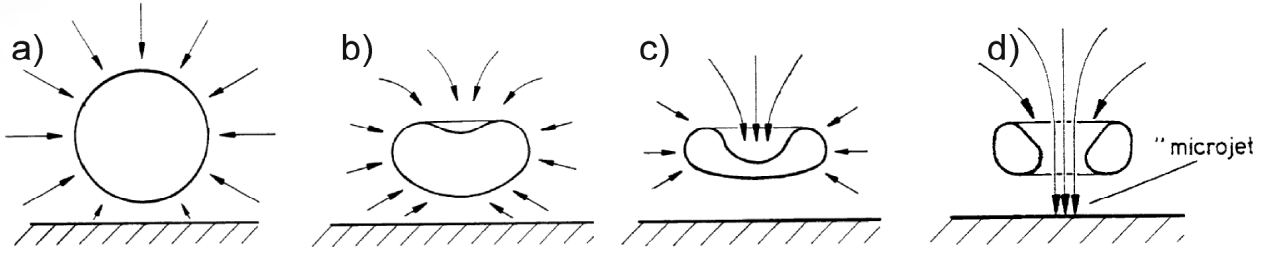

Fig. 1: Sequence of cavitation: a) begin of contraction b)+c) occur of microjet d) Microjet hits the surface [3]

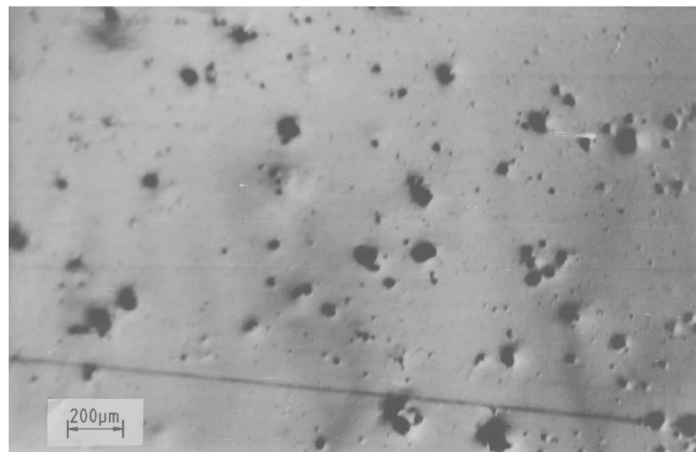

Fig. 2: View on a surface with cavitation dents [3]

To have an indicator, how near the hazard of cavitation is, a value, called Net Positive Suction Head (NPSH) was created. It gives information, as high the reserve is before the fluid evaporates. It is defined in meter fluid head. To know, if a pump can be used in a factory, the manufacturer gives minimal NPSH value (NPSHR, R for required) its pumps need for a cavitation-free work and the purchaser knows which value is available in its complex (NPSHA, A for available). Thus, NPSHA must higher as NPSHR.

In Fig. 4 [5] different ranges of cavitation are shown, the NPSH-value is the reference and plotted on the $x$-axis. The first indication of cavitation is noise. It is detectable with a hydrophone and later, if it is stronger, with an accelerometer at the surface. If the NPSH-value drops, abrasion will begin. That point depends on the impeller material. With decreasing pressure the abrasion increases until to a maximum point. In the near, a further indicator of current cavitation is shown in the diagram, the pump head drop by $3 \%$ as a result of cavitation. It is a standard indicator in pump industry and called NPSH $3 \%$. The important result is: The beginning of cavitation can be shown in a NPSH range where it doesn't damage the pump. By monitoring the current the acceleration amplitude, it will be possible to get information how aggressive the actual working point for the impeller is.

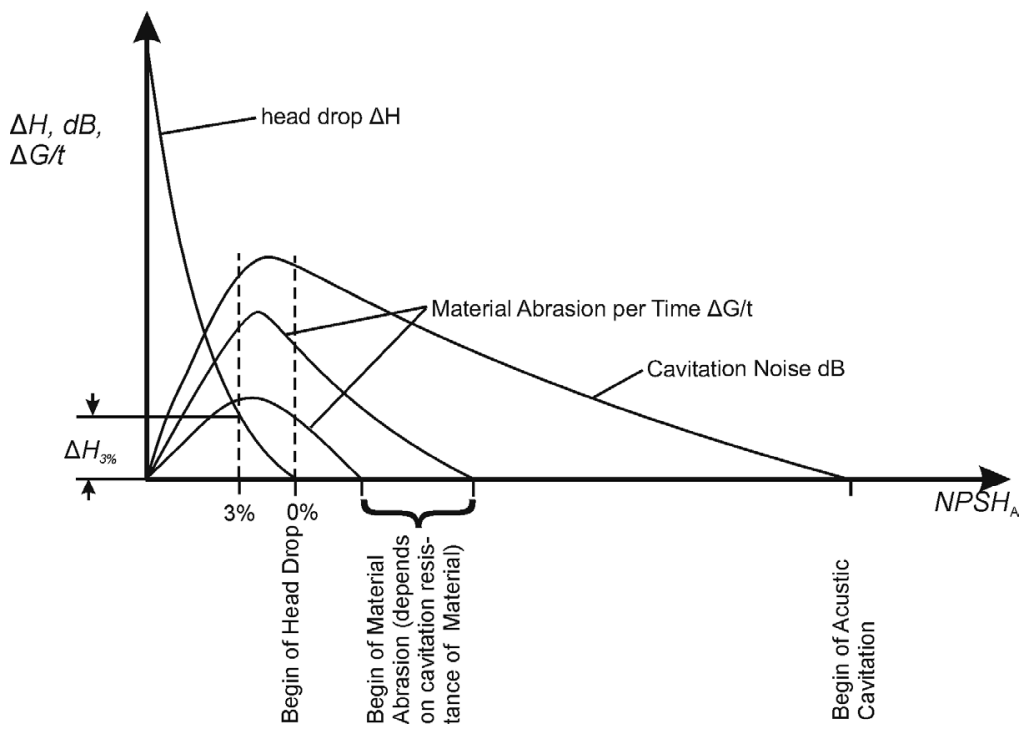

Fig. 3: Head drop, abrasion and cavitation noise over NPSH value [5]. 


\section{Position of cavitation}

Cavitation starts not at all area inside a rotary pump at the same time. At first, the area where it starts must be known to determine the best position for the detector. As described before, the pressure must sink beneath the vapor pressure. This case is in the area where the fluid enters the pump and flows between the blades into the impeller. Here, the flow profile is smaller and the fluid speed higher. Therefore, the pressure is, according to the Bernoulli equation, lower and thus, cavitation starts at these points. It is shown in figure 4 as white ellipses. If the pressure farther decreases or the flow rate increases, the cavitation will be stronger. The bubbles now minimize the flow profile and the fluid speed will increase. It is a reinforcing process. Flow rate and delivery head ease to zero. Micro jets induce vibrations into the impeller and fluid. The detection of structure-borne noise in the impeller can be done by acceleration sensor. The following transferring of the signal out of the pump by radio transmission is necessary because of the galvanic isolation of the rotating part, but it makes the whole electronic very complex. Additional, this realization isn't practicable in industrial application because it is necessary to attach a power supply for the transceiver at the impeller. Another possibility is the using of the suction nozzle which is in the near of the cavitation region. The acoustic waves come directly to the nozzle surface through the water and induce their energy directly in the material. The vibrations can be measured by an acceleration sensor at the surface (see Fig. 5). The used sensor has measuring range from $30 \mathrm{kHz}$ to $2 \mathrm{MHz}$ and can detect the real signal characteristics (Fig. 6 left). The acoustic power of the signal can be calculated, and it is a dimension of the cavitation aggressiveness. An overview of the acoustic power at different NPSH values is given in figure 6 (right) by $n=1500 \mathrm{~min}^{-1}$ and $q_{v}=69 \mathrm{~m}^{3} / \mathrm{h}$. A significant growth of the power is visible at NPSH $=4 \mathrm{~m}$, it is the point where cavitation begins to be destructive.

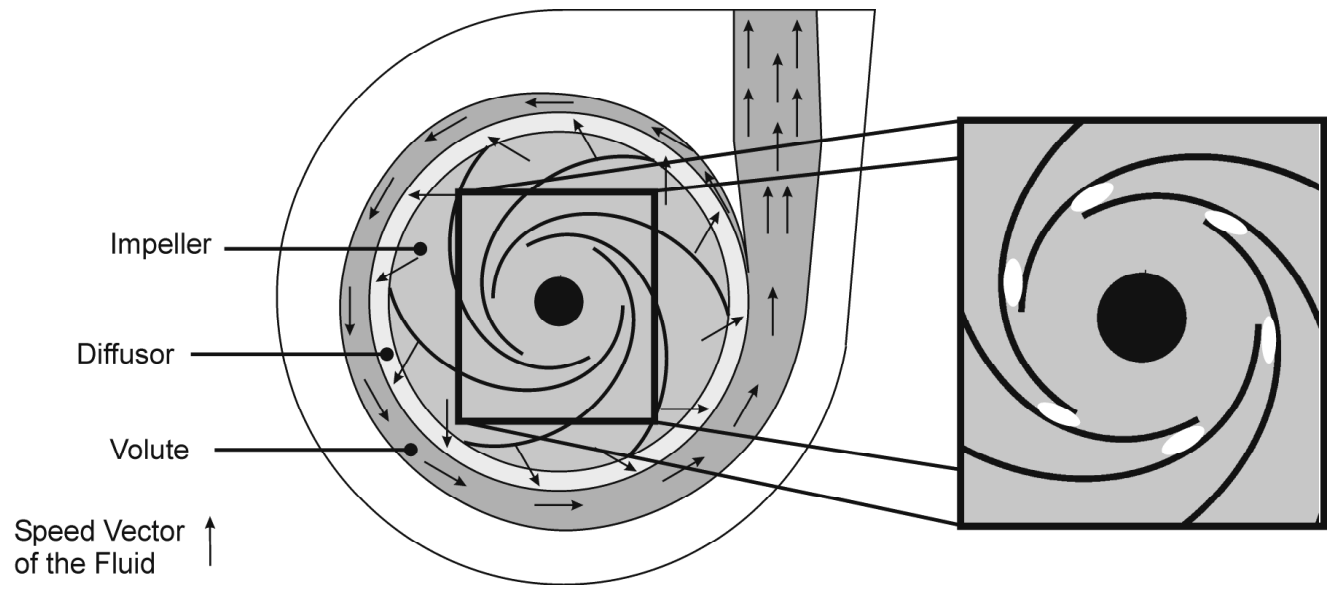

Fig. 4: Sectional view inside a rotary pump with arrows which show the fluid flow. Cavitation starts where the fluid flows through the blades inside the impeller (white areas at right side)

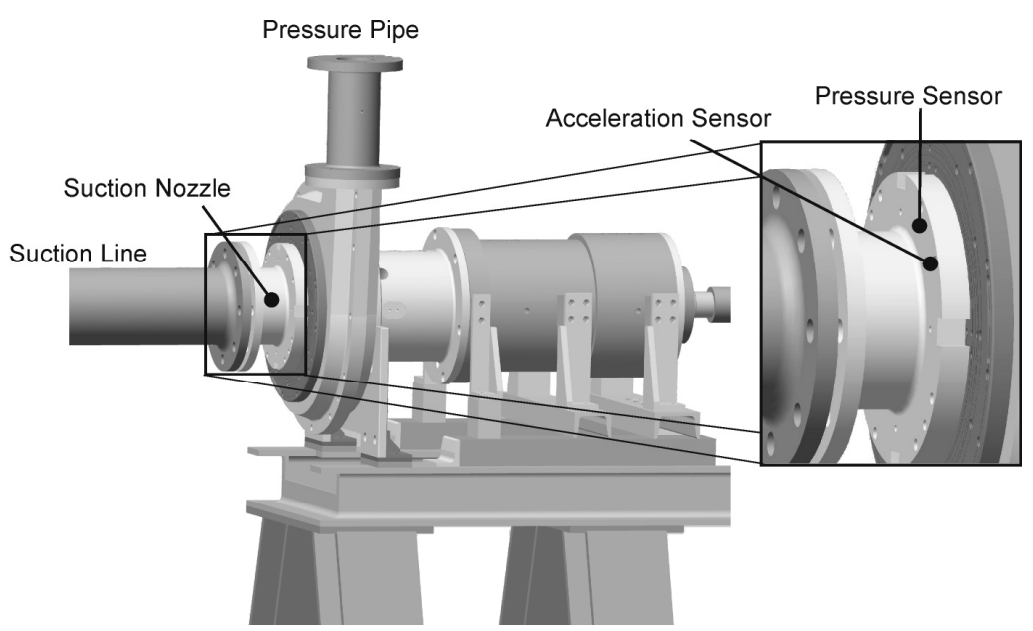

Fig. 5: Sensor positions at the suction nozzle 

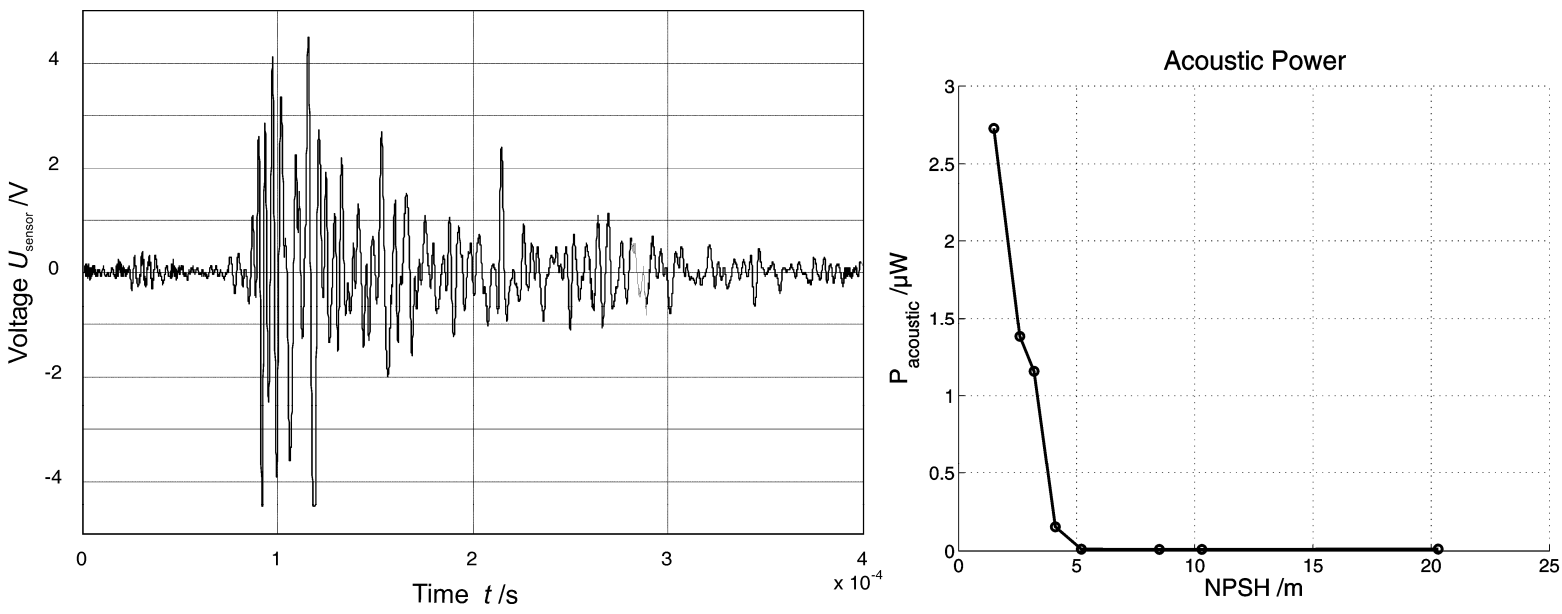

Fig. 6 : Structure-borne noise after a cavitation implosion and the curve of the acoustic power at different NPSH values

In the near of the acceleration sensor already a pressure sensor is integrated which measures the offset pressure at the suction line. The idea is now to use this sensor for cavitation intensity detection and to save the acceleration sensor.

\section{Measurement Results with the Pressure Sensor}

Standard pressure sensors, how measures statistic values up to 15 bar, can't detect such high frequencies as produced by the implosions. So, a signal like this in fig 6 left isn't visible. The used sensor is from manufacturer Kulite ${ }^{\circledR}$ (XTM-190) with a natural frequency of $285 \mathrm{kHz}$. But the task isn't to determine the current cavitation aggressiveness. Instead, it is the goal to make estimation how destructive the actual situation is. Therefore, it is only necessary to find an indicator for it. One solution could be a search for characteristic frequencies or frequency ranges. With these characteristics it will be possible to make an educated presumption and confirms it with a comparison between the acoustic power and the indicator. One of the first results can be seen in figure 7. At the left side is shown a spectrum at a NPSH $=20 \mathrm{~m}$, the pressure is too high for cavitation. In the range from 40 to $200 \mathrm{kHz}$ the amplitude average value is about $-85 \mathrm{dBu}$. At the other side a spectrum of NPSH $=2 \mathrm{~m}$ is shown. At the suction line the spectrum has been changed. It gives a maximum at $85 \mathrm{kHz}$ with amplitude of $-72 \mathrm{dBu}$. Is this maximum specific for cavitation? To investigate it, this frequency range (80 to $100 \mathrm{kHz}$ ) will be enlarged and checked at different NPSH-values. In figure 8 the result is shown. The amplitude at $82 \mathrm{kHz}$, averaged over a range of $\pm 2 \mathrm{kHz}$, is plotted over the $\mathrm{NPSH}$-value. Till $4 \mathrm{~m}$ the amplitude is at $-95 \mathrm{dBu}$. Than, the amplitude growth with decreasing NPSH-values till a maximum by $1,3 \mathrm{~m}$. This fact corresponds to the run of acoustic power curve in figure 3 .
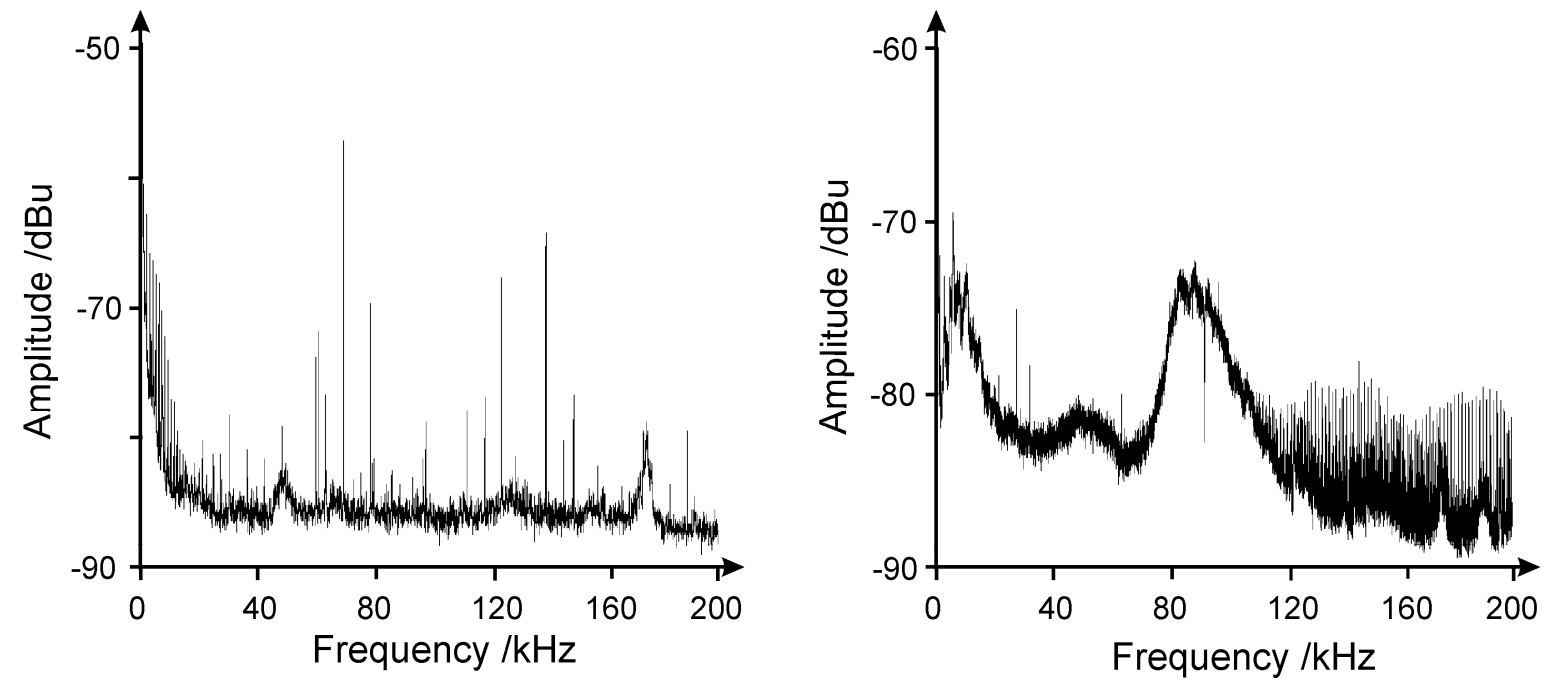

Fig. 7: Spectrum of the sensor signal at NPSH $=20 \mathrm{~m}$ (left) and NPSH $=2 \mathrm{~m}$ (right). 


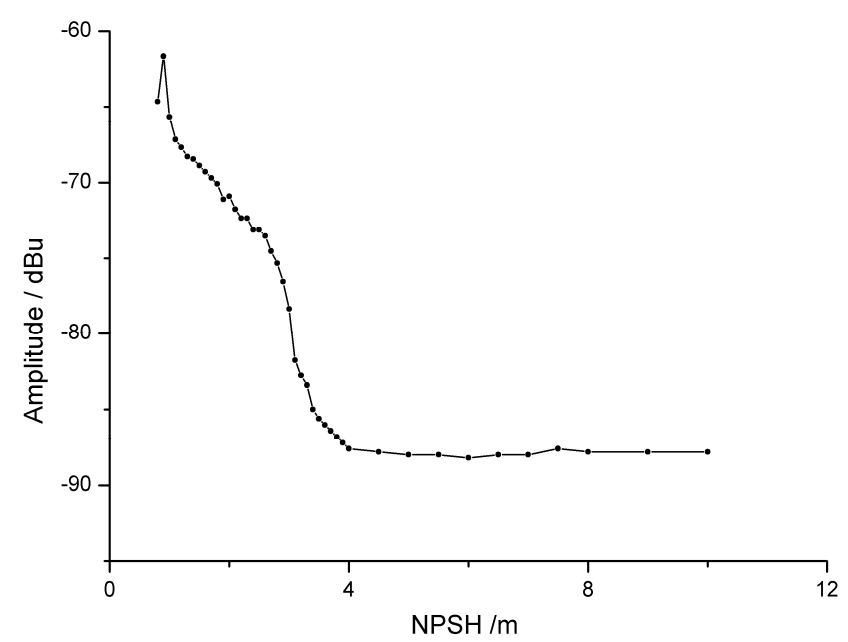

Fig. 8: Sensor output signal over the NPSH value

The presumption is that cavitation induces in one part, perhaps the impeller, vibrations in one of its resonance frequency. This theory would explain this clearly dependence between the acoustic noise and the measured frequency maximum by $82 \mathrm{kHz}$.

Further measurement series showed that, in case of no cavitation, the amplitude is about $-85 \mathrm{dBu}$. The cavitation amplitude rise at $82 \mathrm{kHz}$ always exists but the amplitude varies from series to series about \pm 5 $\mathrm{dBu}$. Thus, it is only possible to make a qualitative conclusion and divide the cavitation in four classes: no cavitation, begin of cavitation, medium cavitation and strong cavitation. This information is important for the sensor system. It can now react and change the rotational speed and/or give an alert to the control room.

\section{Outlook}

The measurement results show that it is possible to detect and classify the cavitation inside pumps with pressure sensors. In further measurement series the whole rotating speed range between $1000 \mathrm{~min}^{-1}$ and $2500 \mathrm{~min}^{-1}$ and at different working points has to be investigate whether the diagnose system works properly and the detection rate is good. The presented method should work at every rotary but the frequency range, where the amplitudes have their maximum, could differ. Probably the natural impeller frequency is the determining point. The presented cavitation detection will be a part of the developed sensor system which was described at the beginning of the paper.

\section{Literatur}

[1] Werner, R.; Werthschützky, R.: Flow Rate Detection by means of pressure difference measurement. In : Proceedings of Sensor 07. Nürnberg, 2007

[2] Werner, R.; Ayllón-Clemente, I.: Werthschützky, Roland. Pressure Transducers as a rotational speed detector for rotodynamic pumps. In: Proposal to Eurosensor09 Dresden, Dresden 2008.

[3] Young, F. Roland; Cavitation.McGraw-Hill Book Company, 1989

[4] Stoffel, B.: Kavitation. Skriptum zur Vorlesung Kavitation. Sommersemester 2005. Darmstadt 2005

[5] Pfleiderer, C.: Petermann, Hartwig. Strömungsmaschinen. Springer Verlag, 7. Auflage 2004 